\title{
ANÁLISE DO TERMO DE PRIMEIRA ORDEM DAS SÉRIES \\ DE MOLODENSKII PARA O PROBLEMA DE VALOR DE \\ CONTORNO DA GEODÉSIA
}

An analysis of First-order Term of the Molodenskii's Series for Geodetic Boundary Value Problem

\author{
VAGNER GONÇALVES FERREIRA \\ SILVIO ROGÉRIO CORREIA DE FREITAS
}

Universidade Federal do Paraná

Curso de Pós-graduação em Ciências Geodésicas

Caixa postal, 19001 - Centro Politécnico - Jardim das Américas

81.531-990 - Curitiba Paraná - Brasil

\{vgf@ufpr.br, sfreitas@ufpr.br\}

\begin{abstract}
RESUMO
Neste trabalho, avaliou-se o termo de primeira ordem das séries de Molodenskii usando as seguintes abordagens: a solução dada pela série de Molodenskii; a solução pelo gradiente vertical; e a solução pela correção de terreno como aproximação do termo $G_{1}$. As duas últimas soluções foram obtidas por Moritz. As duas primeiras soluções mostraram-se coerentes entre si nas condições aqui analisadas. A comparação foi feita em termos de anomalia de altitude de primeira ordem $\zeta_{1}$. Em termos do quase geoide final, o uso da correção de terreno em vez do termo $G_{1}$ mostrou-se eficiente em termos da avaliação realizada com dados independentes; neste caso, 42 referências de nível posicionadas por GPS. A metodologia, os resultados, as discussões, conclusões e recomendações práticas são apresentadas.
\end{abstract}

Palavras-chave: Quase Geóide; Anomalia de Gravidade Ar-livre; Problema de Valor de Contorno da Geodésia.

\section{ABSTRACT}

The main purpose of this paper is to evaluate the first-order term of the Molodenskii series by using different approaches, which approaches are: the solution given by Molodenskii; the solution using the vertical gradient in accordance with Moritz as an 
approximation of $G_{1}$ term; and by using the terrain correction which is an approximation for $G_{1}$. The two first solutions are coherent with each other under the conditions here analyzed. The comparison was made in terms of the first order height anomaly. Considering the final quasi-geoid, the use of terrain correction instead of the $G_{1}$ term was efficient in terms of the evaluation by using independent data; in this case, 42 GPS points on benchmarks. The methodology, results and discussions, conclusions and practical recommendations are presented in the paper.

Keywords: Quasi-Geoid; Free-air Gravity Anomaly; Geodetic Boundary Value Problem

\section{INTRODUÇÃO}

Historicamente, a determinação do campo de gravidade tem sido tratada com o Problema de Valor de Contorno da Geodésia (PVCG), i.e., iniciando-se a partir de medidas na superfície da Terra, e usando-as para determinação de um modelo geoidal, por exemplo. Para Gelederen e Rummel (2001), o PVCG é o ponto de partida para o cálculo do geoide, bem como para a representação do campo de gravidade. Esse pensamento leva à formulação do PVCG, que pode ser entendida, como a determinação de uma função harmônica no exterior de uma superfície de contorno, a partir de funcionais do campo de gravidade conhecidos na superfície de cálculo (anomalias de gravidade, distúrbios de gravidade, diferença de potencial, etc.), resultando na equação integral (HEISKANEN; MORITZ, 1967, p. 298):

$$
-2 \pi T+\iint_{S}\left[T \frac{\partial}{\partial n}\left(\frac{1}{\ell}\right)-\frac{1}{\ell} \frac{\partial T}{\partial n}\right] d S=0
$$

A (1) é a solução da equação de Poisson para $T$ na forma de uma integral; onde $\ell$ é a distância entre o ponto de cálculo e o ponto variável de integração; $T$ representa a diferença entre o potencial da gravidade da Terra real $W$ e o potencial da gravidade da Terra normal $U$ no mesmo ponto; e $n$ é normal a superfície $S$. Para maiores detalhes sugere-se: Kellogg (1929); Moritz (1965); Heiskanen e Moritz (1967); Pick, Pícha e Vyskočil (1973); e Roy (2008).

A linearização da (1) requer uma superfície de aproximação para a superfície de contorno $S$. Se $S$ for o geoide, esta aproximação será referida ao elipsoide (Stokes). Se $S$ é a superfície terrestre, esta aproximação será o teluroide (Molodenskii). As abordagens de Stokes e Molodenskii são as soluções clássicas para a determinação gravimétrica do geoide ou quase geoide, respectivamente. A teoria de Stokes e a teoria de Molodenskii assemelham-se ao terceiro problema de valor de contorno da teoria do potencial (HOFMANN-WELLWNHOF; MORITZ, 2006, pp. 27-29). Estas abordagens produzem alturas geoidais e anomalias de 
altitude, a partir de anomalias de gravidade fornecidas sobre o geoide e a superfície física, respectivamente.

As grandezas e superfícies envolvidas podem ser visualizadas na Figura 1. Com o arranjo desta figura considere-se agora o valor da gravidade $g$ para o ponto $P$ e a gravidade normal $\gamma$ para o ponto $Q$. A anomalia de gravidade no contexto da Teoria de Molodenskii é definida como (HEISKANEN; MORITZ, 1967, p. 293):

$$
\Delta g=g_{P}-\gamma_{Q},
$$

onde $P$ está localizado sobre a superfície terrestre e $Q$ sobre o ponto correspondente a $P$ para o teluroide.

Figura 1 - Altitude normal $H_{P}^{N}$ e anomalia de altitude $\zeta_{P}^{N}$.

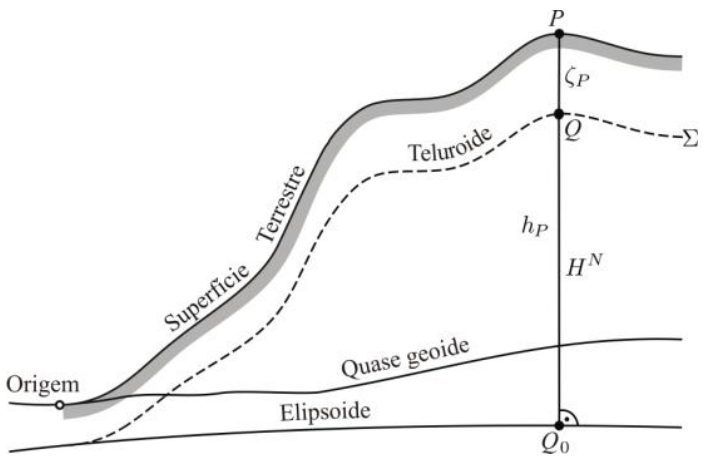

A solução para o PVCG proposta por Molodenskii é baseada em uma série de integrais duplas envolvendo potências de anomalias de gravidade ar-livre e altitudes (MOLODENSKII; EREMEEV; YURKINA, 1962). Neste contexto, o termo de ordem zero $G_{0}$ equivale à própria anomalia de gravidade; o termo de primeira ordem $G_{1}$ é dependente, entre outras variáveis, da variação lateral de altitude (associada à inclinação do terreno) e da anomalia de gravidade. Segundo HofmannWellenhof e Moritz (2006, p. 387), a correção de Molodenskii $\zeta_{1}$ à anomalia de altitude, decorrente da expansão até a primeira ordem $G_{1}$, pode alcançar poucos decímetros, $\zeta_{2}$ e os termos de ordem superiores são frequentemente desprezados. Neste contexto, serão avaliadas estratégias para o cálculo do termo de primeira ordem da série de Molodenskii, associado a $\zeta_{1}$.

Val'ko et al. (2008) compararam duas abordagens para o cálculo dos termos da série de Molodenskii, a saber: aproximação de Molodenskii (MOLODENSKII; 
EREMEEV; YURKINA, 1962); e a da continuação analítica (MORITZ, 1980). Val'ko et al. (2008) ainda avaliaram a utilização da correção de terreno como uma aproximação para o termo de primeira ordem. Os autores concluíram que o uso da correção de terreno $c$ como uma aproximação para o termo $G_{1}$ reduziu a precisão significativamente. No experimento dos mesmos foi observado um aumento de $100 \%$ no desvio padrão para o quase geoide final em relação ao obtido com a determinação rigorosa do primeiro termo da série.

Segundo Moritz (1968), assumindo-se uma relação linear entre o valor da gravidade e a altitude, o termo $G_{1}$ das séries de Molodenskii equivale à correção de terreno. Sanchéz (2003) concluiu que devido a esta correlação com a altitude, as anomalias de Faye ( $\Delta g+c$, sendo $\Delta g$ a anomalia ar livre) são os valores de limite mais adequados para solucionar o PVCG. Sideris e Schwarz (1986) complementam afirmando que o uso da anomalia de Faye é uma alternativa conveniente para a aproximação da solução de Molodenskii, principalmente porque a correção topográfica pode ser determinada de forma precisa e rápida com a técnica Fast Fourier Transform (FFT).

Muitos trabalhos foram publicados abordando o tema das séries de Molodenskii, entre eles podem ser destacados: Monin (1965), Sansò (1978), Shepperd (1982), Velkoborsky (1969), Yeremeev e Yurkina (1970 e 1974), Sideris (1990), Amod e Merry (2002).

\section{AS SOLUÇÕES PARA A SÉRIE DE MOLODENSKII}

\subsection{Abordagem de Molodenskii}

De acordo com Molodenskii, Eremeev e Yurkina (1962, capítulo V) a (1) é linearizada usando o teluroide $\Sigma$. O ponto essencial, entretanto, é que para a (1) a integração sobre a superfície desconhecida $S$ pode ser substituida por uma integração sobre a superfície conhecida $\Sigma$. A avaliação de $\partial T / \partial n$ proposta por Molodenskii é um pouco afanosa. Assim, aqui segue-se a solução mais conveniente encontrada em Heiskanen e Moritz (1967, p. 298):

$$
\frac{\partial T}{\partial n}=\left[-\Delta g+\frac{1}{\gamma} \frac{\partial \gamma}{\partial h} T+\gamma\left(\xi \tan \beta_{x}+\eta \tan \beta_{y}\right)\right] \cos \beta,
$$

onde $\tan \beta_{x}=\frac{\partial h}{\partial x}, \tan \beta_{y}=\frac{\partial h}{\partial y}$, e $\tan ^{2} \beta=\tan ^{2} \beta_{x}+\tan ^{2} \beta_{y} . \beta_{x}$ é o ângulo de inclinação do perfil do terreno na direção norte-sul, similarmente, $\beta_{y}$ é a inclinação para um perfil leste-oeste, e $\beta$ é o ângulo máximo de inclinação do terreno. $\xi$ e $\eta$ são as componentes do desvio da vertical. 
$\mathrm{Na}$ aproximação esférica, limitando as séries para os dois primeiros termos, o potencial anômalo é dado por Moritz (1980) como:

$$
T=\frac{R}{4 \pi} \iint_{\Sigma}\left(G_{0}+G_{1}+\cdots\right) S(\psi) d \Sigma,
$$

onde $S(\psi)$ é a função esférica de Stokes para o argumento $\psi$, que é a distância esférica entre o ponto de cálculo e o ponto de integração. A função esférica de Stokes tem a expressão (HEISKANEN; MORITZ, 1967, p. 94):

$$
S(\psi)=\frac{1}{\operatorname{sen}\left(\frac{\psi}{2}\right)}-6 \operatorname{sen}\left(\frac{\psi}{2}\right)+1-5 \cos \psi-3 \cos \psi \ln \left[\operatorname{sen}\left(\frac{\psi}{2}\right)+\operatorname{sen}^{2}\left(\frac{\psi}{2}\right)\right]
$$

sendo

$$
\operatorname{sen}^{2}\left(\frac{\psi}{2}\right)=\operatorname{sen}^{2}\left(\frac{\varphi_{P}-\varphi}{2}\right)+\operatorname{sen}^{2}\left(\frac{\lambda_{P}-\lambda}{2}\right) \cos \varphi_{P} \cos \varphi
$$

Os termos $G_{0}$ e $G_{1}$ são dados em Heiskanen e Moritz (1967, seção 8.7) na forma:

$$
\begin{gathered}
G_{0}=\Delta g \\
G_{1}^{S M}=\frac{R^{2}}{2 \pi} \iint_{\sigma} \frac{H-H_{P}}{\ell^{3}}\left(\Delta g+\frac{3 \gamma}{2 R} \zeta_{0}\right) d \sigma .
\end{gathered}
$$

Em que $H_{P}$ e $H$ são as altitudes normais para o ponto de cálculo e o ponto de integração, $\gamma$ é a gravidade normal calculada para o teluroide e $\zeta_{0}$ é a anomalia de altitude aproximada calculada a partir de $G_{0}$. Uma aproximação, usualmente adotada para a (8), é dada por (HEISKANEN; MORITZ, 1967, p. 310):

$$
G_{1}^{S M} \approx \frac{R^{2}}{2 \pi} \iint_{\sigma} \frac{H-H_{P}}{\ell^{3}} \Delta g d \sigma .
$$

Note que $G_{1}$ na (9) depende somente da inclinação do terreno e da anomalia da gravidade. A distância Euclidiana $\ell$ é dada por (HEISKANEN; MORITZ 1967, p. 38): 


$$
\ell=2 \operatorname{Rsen} \frac{\psi}{2}
$$

De acordo com Moritz (1968), asumindo uma correlação linear entre anomalias de gravidade com a altitude, o termo $G_{1}^{S M}$ (equação 9) pode ser aproximado pela correção de terreno $c$. Para maiores detalhes, sugere-se Moritz (1968), Sideris (1990) e Torge (2001).

O resultado para a (4) mostrado em termos de anomalias de altitude, após a aplicação da fórmula de Bruns em aproximação linear, $\zeta_{P}=T_{P} / \gamma_{Q}$ (HEISKANEN; MORITZ, 1967, p. 306), toma a forma:

$$
\zeta=\zeta_{0}+\zeta_{1}+\cdots
$$

Para o cálculo do termo $\zeta_{1}^{\text {SM }}$ aplica-se o termo $G_{1}^{S M}$ da (8) na (4) juntamente com a fórmula de Bruns. Note que para o uso da (8) deve-se avaliar a priori o termo $\zeta_{0}$. Este termo, por sua vez, depende somente do termo $G_{0}$ dado pela (7) aplicado isoladamente na (4). Sendo assim, avalia-se a seguinte integral:

$$
\zeta_{1}^{S M}=\frac{R}{4 \pi \gamma} \iint_{\Sigma} G_{1}^{S M} S(\psi) d \Sigma .
$$

A correção de terreno ( $c$ ) para o termo $G_{1}$ dado pela (9), que por sua vez é uma aproximação para a (8), é empregada na (4) obtendo assim a anomalia de altura $\zeta_{1}^{c}$ na forma:

$$
\zeta_{1}^{c}=\frac{R}{4 \pi \gamma} \iint_{\Sigma} \underbrace{\left\{\frac{1}{2} k \rho \int_{-\infty}^{+\infty} \int_{-\infty}^{+\infty} \frac{\left(H-H_{P}\right)^{2}}{\left[\left(x-x_{P}\right)^{2}+\left(y-y_{P}\right)^{2}\right]^{3 / 2}} d x d y\right\}}_{\text {correção de terreno }} S(\psi) d \Sigma .
$$

Sendo $k$ a constante gravitacional $\left(6,67 \times 10^{-11} \mathrm{~m}^{3} \mathrm{~kg}^{-1} \mathrm{~s}^{-2}\right)$ e $\rho$ a densidade crustal média $\left(2,67 \mathrm{gcm}^{-3}\right)$. Detalhes sobre a correção de terreno $c$ podem ser encontrados em Torge (2001, p. 265).

\subsection{A Solução Gradiente}

Um método importante para determinar a anomalia de altitude é fornecido por Moritz (1980, seção 45) denominada como "solução gradiente" (do inglês gradient solution). A solução para o problema simples de Molodenskii por meio da solução gradiente é obtida a partir da integral:

Bol. Ciênc. Geod., sec. Artigos, Curitiba, v. 16, nº 4, p.557-574, out-dez, 2010. 
Ferreira, V. G. ; Freitas, S. R. C.

$$
T=\frac{R}{4 \pi} \iint_{\Sigma}\left(G_{0}+G_{1}+\cdots\right) S(\psi) d \Sigma .
$$

Usando a "Continuação Analítica" (Analitical Continuation), pode-se calcular o termo $G_{1}$ como (MORITZ, 1980, p. 387):

$$
G_{1}^{S G}=-\frac{\partial \Delta g}{\partial r}\left(H-H_{P}\right)
$$

Sendo o gradiente de anomalia de gravidade dado por (PICK; PÍCHA; VYSKOČIL, 1973, p. 313):

$$
\left(\frac{\partial \Delta g}{\partial r}\right)_{P}=\frac{R^{2}}{2 \pi} \iint_{\sigma} \frac{\Delta g-\Delta g_{P}}{\ell^{3}} d \sigma .
$$

em que $\Delta g_{P}$ e $\Delta g$ representam a anomalia de gravidade ar-livre no ponto de cálculo e no ponto de integração, respectivamente. $\mathrm{O}$ termo $G_{0}$ é idêntico como na abordagem de Molodenskii (equação 7).

A anomalia de altitude, empregando a solução gradiente, neste caso usando o gradiente vertical da anomalia de gravidade ar-livre, pode ser resumido por:

$$
\zeta_{1}^{S G}=\frac{R}{4 \pi \gamma} \iint_{\Sigma}\left[-\left(H-H_{P}\right) \frac{\partial \Delta g}{\partial r}\right] S(\psi) d \Sigma
$$

\section{EXPERIMENTOS NUMÉRICOS}

\subsection{Dos Dados Utilizados}

A área limitada pelos paralelos $\varphi=-26^{\circ}, \varphi=-23^{\circ}$ e meridianos $\lambda=-54^{\circ} \mathrm{e}$ $\lambda=-49^{\circ}$ foi escolhida para cálculos numéricos e comparações. As razões que levaram a esta escolha esta no raio de integração empregado para os cálculos, neste caso $\psi=1^{\circ}$. Os dados de entrada, valores de gravidade observados associados com altitudes estão disponíveis em uma área limitada pelos paralelos $\varphi=-27^{\circ}$, $\varphi=-22^{\circ}$ e meridianos $\lambda=-55^{\circ}$ e $\lambda=-48^{\circ}$. Esta área contém cerca de 4.111 pontos gravimétricos conforme mostra a Figura 2. O acervo de dados gravimétricos é proveniente do banco de dados da Escola Politécnica da Universidade de São Paulo (POLI-USP) (BLITZKOW, 2006). Nesta mesma área estão disponibilizadas cerca de 110 referências de nível (RNs) ocupadas com o GPS (Global Positioning System) pertencentes ao Departamento de Geofísica, Laboratório de Geodésia do 
Instituto Astronômico e Geofísico da Universidade de São Paulo (IAG-USP) (SÁ, 2007).

Empregou-se o modelo digital de elevação (MDE) SRTM-3 (RODRIGUEZ et al., 2005) com 3" x3" de resolução espacial, conforme Figura 3, para o processo de estimação de anomalias de gravidade conforme Featherstone e Kirby (2000) e para o cálculo da correção do tereno. Originalmente, o SRTM-3 têm suas altitudes referenciadas ao Earth Gravity Model 96 [EGM96, (LEMOINE et al., 1998)] de acordo com Rodriguez et al., (2005) e Farr et al., (2007). No contexto deste trabalho, o MDE foi referenciado ao Earth Gravity Model 2008 [EGM2008, (PAVLIS et al., 2008)] em termos de altitudes normais $\left(H^{N}\right)$ para o cálculo das anomalias de gravidade empregando a (2). Resumidamente, somaram-se as alturas geoidais advindas do EGM96 as altitudes do SRTM, e em seguida, subtraíram-se destas as anomalias de altitude advindas do EM2008.

Figura 2 - Estações gravimétricas e GPS/RNs para a área de estudos.

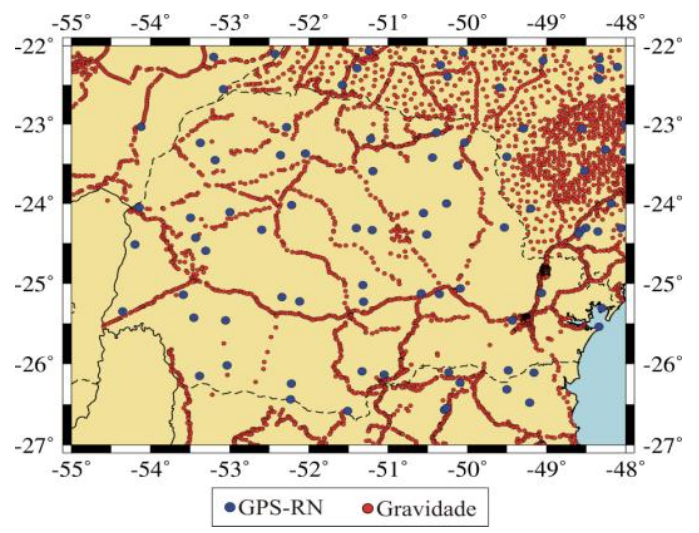

Para todos os cálculos, as anomalias de gravidade ar-livre residual foram calculados a partir do EGM2008 até o grau 2190 e ordem 2159 na forma:

$$
\Delta g_{\text {res }}=\Delta g-\Delta g_{E G M} \text {. }
$$

A anomalia de gravidade proveniente do EGM2008 $\left(\Delta g_{E G M}\right)$ foi avaliada sobre a superfície terrestre, para detalhes consultar Barthelmes (2009, p. 16, equação 101). Neste trabalho não se considerou o efeito das altas frequências do campo de gravidade.

Os valores das anomalias de gravidade ar-livre no contexto da teoria de Molodenskii podem ser visualizados na Figura 4. Todas estas informações foram geradas a partir da distribuição de dados gravimétricos para a região mostrada na 
Ferreira, V. G. ; Freitas, S. R. C.

Figura 2, onde todas as interpolações foram geradas em termos das anomalias Bouguer, pois estas, em princípio, não são correlacionadas com a altitude.

Figura 3 - Topografia da região de estudos a partir do SRTM-3 reamostrado.

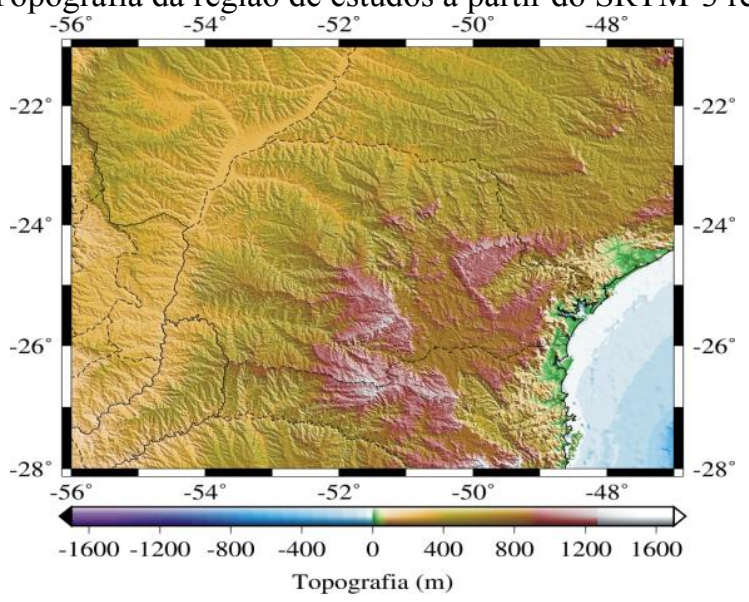

Figura 4 - Anomalias de gravidade ar-livre.

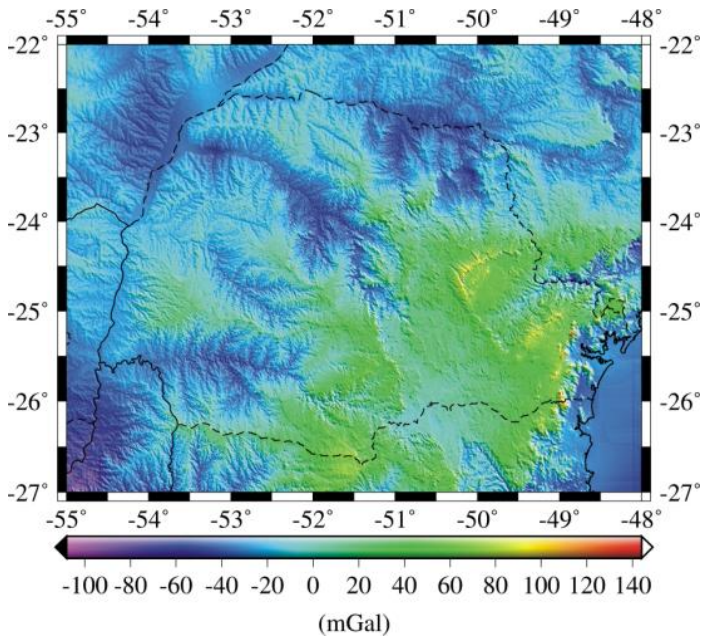

A estratégia para a predição dos valores de anomalias de gravidade tem como base o método de Featherstone e Kirby (2000) que minimiza efeitos de mascaramento (aliasing) devido ao processo de amostragem irregular dos valores de gravidade. 


\subsection{Dos Resultados Obtidos}

Os resultados para o termo $G_{1}^{S M}$, advindos da (8), e para o gradiente vertical de anomalia de gravidade ar-livre, advindos da (16), foram obtidos aplicando as anomalias de gravidade ar-livre residuais. Para a solução destas integrais aplicou-se integração numérica clássica com base no método dos blocos formado por coordenadas geográficas conforme Heiskanen e Moritz (1967, p. 119). Outra forma de se avaliar estas integrais pode ser encontrada em Sideris e Schwarz (1986) que fazem uso da técnica FFT. Uma comparação quantitativa entre a integração numérica direta e a solução no domínio das frequências pode ser encontrada em Hofmann-Wellenhof e Moritz (2006, p. 387, Tabela 11.4). A integração numérica foi realizada com uma distância geocêntrica de até $\psi_{\text {máx }}=1^{\circ}$, conforme comentando na seção anterior. Não se realizou aqui a busca de um raio ótimo de integração. Para o termo $G_{1}$ obtido com a (8) tem-se o resultado mostrado na Figura 5 e o gradiente vertical da (16) é mostrado na Figura 6. Na Figura 7 apresenta-se a correção de terreno para a região de estudos.

Figura 5 - O termo $G_{1}$ calculado a partir da abordagem de Molodenskii.

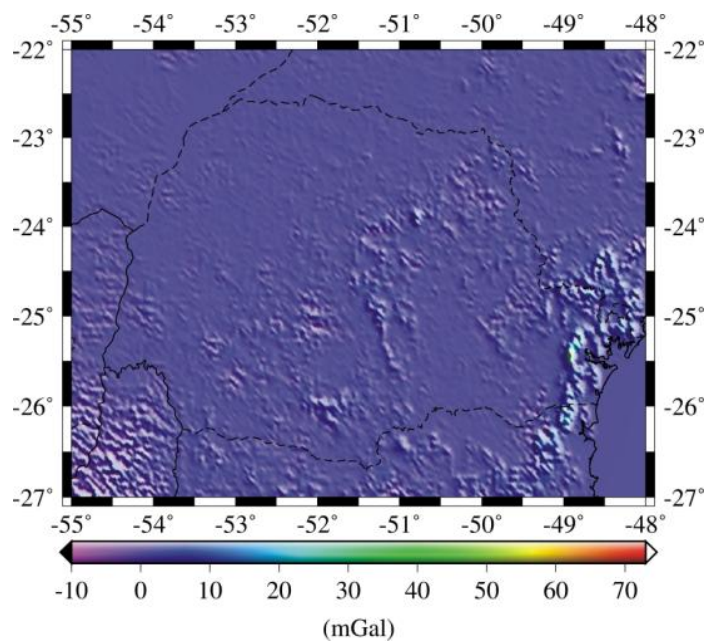

Note-se que estas duas abordagens representadas nas Figuras 5 e 6 não podem ser comparadas diretamente. A diferença formal entre elas, após algumas simplificações é dada por:

$$
G_{1}^{S M}-G_{1}^{S G} \approx \frac{R^{2}}{2 \pi} \iint_{\sigma} \frac{H-H_{P}}{\ell^{3}} \Delta g_{P} d \sigma .
$$


Observe-se que a anomalia de gravidade no integrando da (19) refere-se ao ponto onde se deseja determinar o quase geoide, enquanto que no integrando da (8) refere-se ao elemento de integração $d \sigma$. Apesar disso, pode-se comparar o efeito em termos de anomalia de altitude. Na Tabela 1 tem-se a comparação das diversas soluções abordadas neste trabalho, e respectivas estatísticas.

Figura 6 - O gradiente vertical para a anomalia de gravidade ar-livre residual em Eötvös (E) usando continuação analítica.

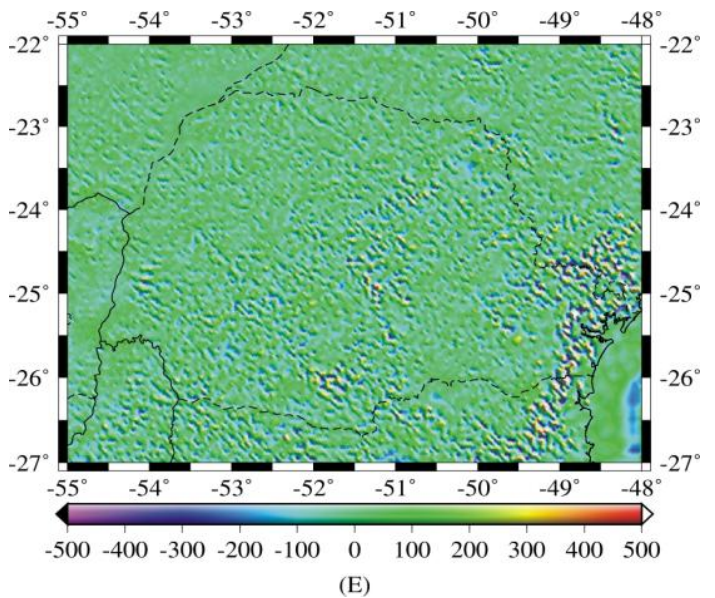

Tabela 1 - Estatísticas básicas para as anomalias de altitude de primeira ordem por diversas abordagens.

\begin{tabular}{c|c|c|c}
\hline Estatística & $\zeta_{1}^{S M}(\mathrm{~m})$ & $\zeta_{1}^{c}(\mathrm{~m})$ & $\zeta_{1}^{S G}(\mathrm{~m})$ \\
\hline Mínimo & 0,053 & 0,058 & 0,053 \\
Máximo & 0,265 & 0,210 & 0,270 \\
Amplitude & 0,212 & 0,152 & 0,217 \\
Média & 0,126 & 0,125 & 0,125 \\
Desvio padrão & 0,049 & 0,032 & 0,048 \\
Erro médio quadrático & 0,135 & 0,129 & 0,134 \\
\hline
\end{tabular}

Comparando os resultados para $\zeta_{1}^{S M}$ e $\zeta_{1}^{c}$ (colunas 2 e 3 da tabela 1) nota-se pequena diferença entre os mesmos, tomando-se o resultado da (12) como parâmetro, o uso da correção de terreno para o termo $G_{1}^{S M}$ (equação 8) pode conduzir a diferenças significativas no resultado final (quase geoide). Outro fato importante é que a correção de terreno para a área de estudos foi positiva, como deveria de ser, pois a mesma foi determinada por aproximação planar, justificando assim, os valores positivos para $\zeta_{1}^{c}$. Para o termo $G_{1}^{S M}$ observou-se valores 
positivos e negativos, obviamente devidos aos valores das anomalias de gravidade ar-livre residuais.

Figura 7 - Correção de terreno para a área de estudos.

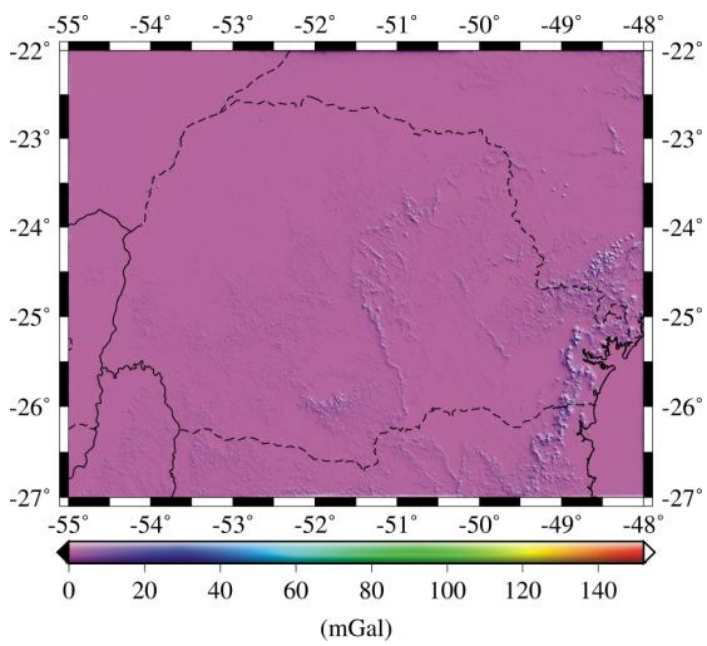

Comparando-se os resultados para $\zeta_{1}^{S M}$ e $\zeta_{1}^{S G}$ na Tabela 1 (colunas 2 e 4, respectivamente) não há diferença significativa entre as soluções. A diferença entre ambas é menor que $1 \mathrm{~cm}$ para o termo de primeira ordem das séries de Molodenskii. No entanto, para o cálculo dos demais termos da série de Molodenskii, é viável o emprego da solução gradiente, em que a mesma pode ser avaliada recursivamente (MORITZ, 1980, p. 386).

Na Figura 8 pode-se observar a dispersão da anomalia ar-livre residual $\Delta g_{\text {res }}$ em relação à altitude, bem como a correção de terreno $c$ e o termo $G_{1}$ dado pelas equações (16 e 15). Constata-se que o termo $G_{1}$ apresenta maior correlação com a altitude do que a própria anomalia ar-livre residual $\Delta g_{\text {res }}$ e do que correção de terreno $c$. Ao subtrair os longos comprimentos de ondas com o uso do EGM2008 (equação 18), estes absorveram aproximadamente $64 \%$ do efeito da altitude sobre as anomalias ar-livre. O termo $G_{1}$ apresenta correlação com a altitude de $40 \%$, que provavelmente decorre do seu cálculo ser efetuado com a (15). O termo $G_{1}$ tem a conotação de um gradiente de anomalia, que é resolvido separadamente e operado posteriormente por uma diferença de altitude usualmente pequena em relação à altitude do ponto. 
Figura 8 - Anomalia ar-livre residual, correção de terreno e o termo $G_{1}$.
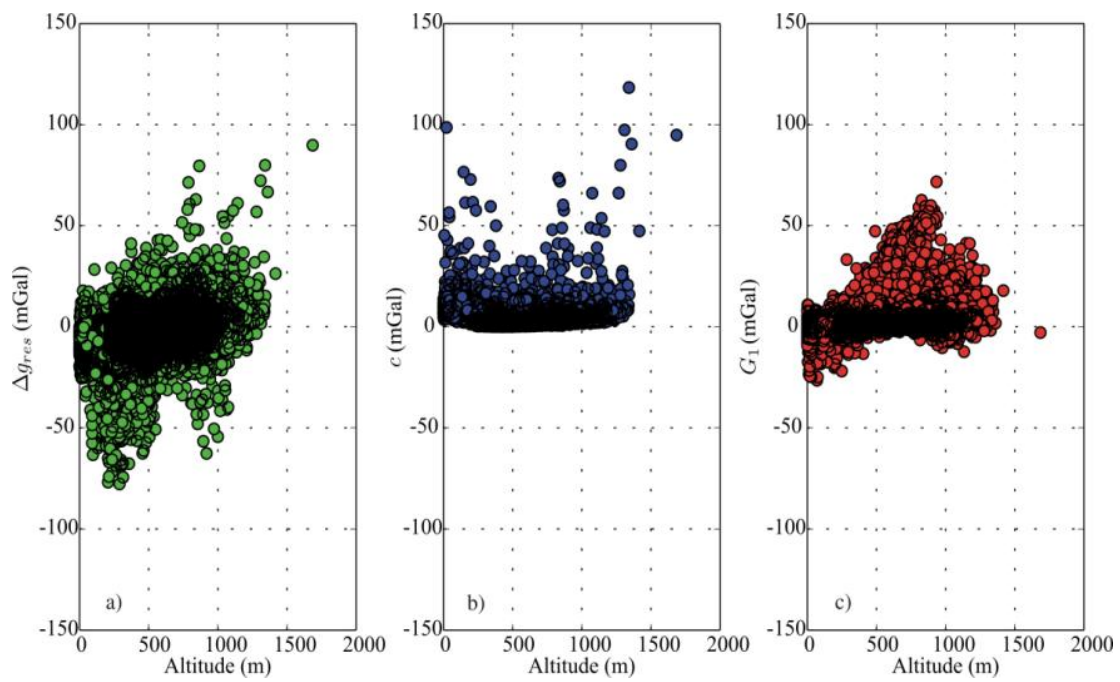

Sideris e Schwarz (1985) interpretaram as diferenças entre $\zeta_{1}^{S M}$ e $\zeta_{1}^{c}$, em termos de valores máximos, como indicadoras da variação da densidade das massas $\rho$. De fato, o sinal gravimétrico das anomalias reflete também a densidade relativa das massas na região. Para a correção de terreno empregou-se o valor padrão $\rho_{0}=2,67 \mathrm{gcm}^{-3}$, e as diferenças podem resultar de densidade variável das massas anômalas para a região de estudos. No entanto, em outras condições, o uso da correção de terreno pode decrescer significativamente a precisão. Em uma determinação rigorosa, o termo $G_{1}$ difere efetivamente da correção de terreno. Esta correção em aproximação planar é sempre positiva. No entanto, o termo $G_{1}$ pode assumir valores negativos e sua magnitude, em geral, são maiores que a correção de terreno (comparar as Figuras 5 e 7, ver também a Figura 8).

Com as soluções compatíveis entre si, considerando a Tabela 1, calculou-se o quase geoide final para a área de estudos, cujos resultados são apresentados no domínio do espaço nas Figuras 9 e 10. A Figura 9 mostra o quase geoide dado pela solução gradiente para o cálculo do termo $G_{1}$. A Figura 10 mostra o quase geoide final empregando-se a correção de terreno como uma aproximação para o termo $G_{1}$. A avaliação com dados independentes, 42 RNs posicionadas por GPS pode ser visualizada na Tabela 2. Ressalta-se que não foi realizada a compatibilização do quase geoide final sobre os pontos de controle, de tal modo que a avaliação aqui apresentada não considera a componente "sistemática" entre o quase geoide calculado e a origem das altitudes das RNs empregadas para avaliação. Note ainda 
nas Figuras 9 e 10 o histograma da avaliação do quase geoide final, percebe-se então que a frequência das discrepâncias é praticamente idêntica em ambas as soluções.

Figura 9 - Quase geoide com base no termo $G_{1}$ empregando a solução gradiente.

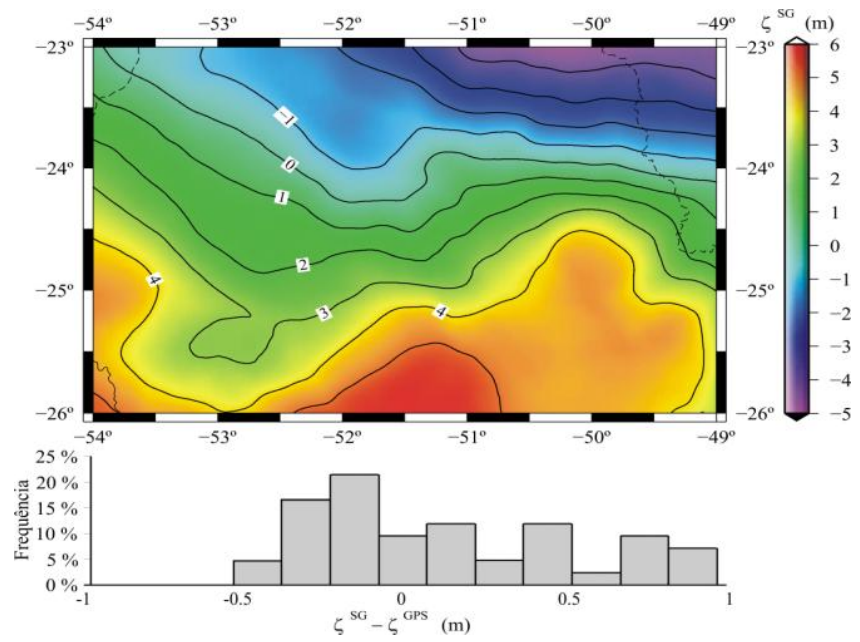

Figura 10 - Quase geoide com base no termo $G_{1}$ aproximado pela correção de terreno.

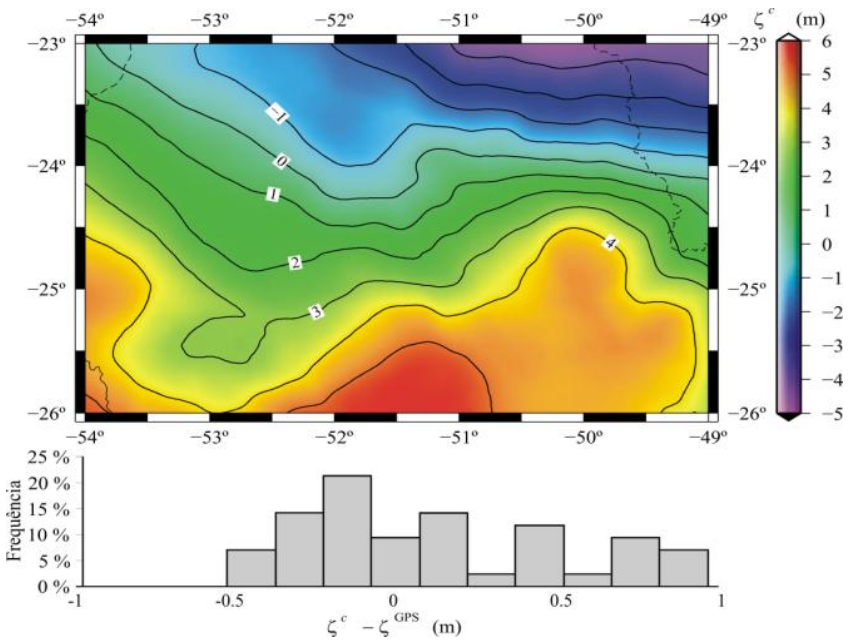

Bol. Ciênc. Geod., sec. Artigos, Curitiba, v. 16, nº 4, p.557-574, out-dez, 2010. 
Tabela 2 - Estatísticas básicas entre as anomalias de altura interpoladas a partir do modelo gravimétrico e 42 pontos GPS/RNs. Para a última coluna, o termo $G_{1}$ foi aproximado pela correção de terreno.

\begin{tabular}{c|c|c}
\hline Estatística & $\zeta^{S G}-\zeta^{G P S}(\mathrm{~m})$ & $\zeta^{c}-\zeta^{G P S}(\mathrm{~m})$ \\
\hline Mínimo & $-0,536$ & $-0,541$ \\
Máximo & 0,972 & 0,958 \\
Média & 0,125 & 0,111 \\
Desvio padrão & 0,429 & 0,429 \\
Erro médio quadrático & 0,447 & 0,443 \\
\hline
\end{tabular}

\section{CONCLUSÕES E RECOMENDAÇÕES}

Do ponto de vista teórico, objetivando calcular o quase geoide com precisão ao nível do centímetro, o termo $G_{1}$ deve ser determinado de forma rigorosa empregando-se a (8) ou a (15). Os resultados em termos de quase geoide final são otimistas, mostrando que a correção de terreno tem fundamentos para ser usada como aproximação para o termo $G_{1}$. No entanto, o fato de se ter utilizado GPS sobre as RNs, no contexto das altitudes brasileiras para avaliar estes modelos, não se pode afirmar qual o quase geoide mais preciso. Deve-se ter em mente que estas altitudes são desprovidas da componente relativa ao campo de gravidade real, i.e., números geopotenciais.

Uma explicação preliminar pode ser em termos da variabilidade irregular da anomalia de gravidade ar-livre na região. Considerando que se utilizou a anomalia ar-livre residual, e esta apresenta uma moderada dependência com a altitude, justificando o uso da correção de terreno para o termo $G_{1}$. No entanto, a correção de terreno aparenta ser mais suave (Figura 8.b), o que é consistente com a sua função, remover o efeito das irregularidades da topografia em relação ao platô de Bouguer, o que é apropriado para o problema de Molodenskii. Recomenda-se extrair as componentes de alta frequência do campo de gravidade na (18) utilizando-se informações da topografia obtendo, neste caso, um sinal residual que é um campo mais suave e apropriado à determinação do quase geoide, além do que atenua efeitos como o da variabilidade da densidade das massas anômalas para a região.

$\mathrm{Na}$ Tabela 1, observa-se que o termo de primeira ordem para as séries de Molodenskii é da ordem do decímetro, tornando-se neste caso necessário o cálculo dos demais termos da série. Uma opção é usar a solução gradiente, pois a mesma pode ser obtida de forma recursiva.

\section{AGRADECIMENTOS:}

Ao Professor Dr. Denizar Blitzkow pelo fornecimento de dados gravimétricos terrestres utilizados neste trabalho. Ao Professor Nelsi Côgo de Sá pelo 
fornecimento de dados GPS sobre RNs utilizados para avaliação dos modelos. Ao CNPq processos 560796/2008-0 e 301797/2008-0, CAPES, PROBRAL (CAPES/DAAD) projeto n. 228/06 processo BEX4157/08-7. Aos revisores e editores pelas críticas construtivas.

\section{REFERÊNCIAS BIBLIOGRÁFICAS}

AMOD, A.; MERRY, C. L. A note on the Molodensky G1 term. Bulletin of the International Geoid Service, n. 12, p. 62-80, 2002.

BARTHELMES, F. Calculation from spherical harmonic models. Pottsdam: GeoForschungsZentrum, 2009. 32 p. Relatório técnico-científico STR09/02.

BLITZKOW, D. Banco de dados gravimétricos. Departamento de Engenharia de Transportes, Escola Politécnica da Universidade de São Paulo. São Paulo. Comunicação pessoal em 2006.

FARR, T. G.; ROSEN, P. A.; CARO, E.; CRIPPEN, R.; DUREN, R.; HENSLEY, S.; KOBRICK, M.; PALLER, M.; RODRIGUEZ, E.; ROTH, L.; SEAL, D.; SHAFFER, S.; SHIMADA, J.; UMLAND, J.; WERNER, M.; OSKIN, M.; BURBANK, D.; ALSDORF, D. The Shuttle Radar Topography Mission, Reviews of Geophysics, vol. 45, p. 1-33, 2007.

FEATHERSTONE, W. E.; KIRBY, J. F. The reduction of aliasing in gravity anomalies and geoid heights using digital terrain data. Geophysical Journal International, v. 141, n. 1, p. 204-212, 2000.

GELEDEREN, M. Van; RUMMEL, R. The solution of general geodetic boundary value problem by least squares. Journal of Geodesy, v. 75, n. 1, p. 1-11, maio 2001.

HEISKANEN, W. A.; MORITZ, H. Physical geodesy. San Francisco and London: W. H. Freeman and Company, 1967.

HOFMANN-WELLWNHOF, B.; MORITZ, H. Physical geodesy. 2. Viena: Springer, 2006.

JAMUR, K. P.; FERREIRA, V. G.; de FREITAS, S. R. C. Avaliação do Earth Gravity Model 2008 (EGM2008) no contexto das altitudes do sistema geodésico brasileiro. In: Colóquio Brasileiro de Ciências Geodésicas, 6, 2009, Curitiba. 1 CD-ROM.

KELLOGG, O. D. Foundations of potential theory. Cambridge: Verlag von Julius Springer, 1929.

LEMOINE, F. G.; KENYON, S. C.; FACTOR, R. G.; TRIMMER, R. G.; PAVLIS, N. K.; CHINN, D. S.; COX, C. M.; KLOSKO, S. M.; LUTHCKE, S. R.; TORRENCE, M. M.; WANG, Y. M.; WILLIAMSON, R. G.; PAVLIS, E. C.; RAPP, R. H.; OLSON, T. R. The development of the joint NASA - GSFC and the NIMA geopotential model EGM96. NASA/TP - 206861. Goddard Space Flight Center, Greenbelt, 1998.

MOLODENSKII, M. S.; EREMEEV,V. F.; YURKINA, M. I. Methods for study of the external gravitational field and figure of the earth. Jerusalém: Israel 
Program for Scientific Translations, 1962.

MONIN, I. F. Solution of the M. S. Molodenskii integral equation defining the figure of the Earth's physical surface with allowange for third-order terms. Soviet Astronomy, v. 9, n. 1, p. 141-145, 1965.

MORITZ, H. The boundary value problem of physical geodesy. International Association of Geodesy, Helsinki: Publ. Isostat. Inst., 1965.

MORITZ, H. On the use of terrain correction in solving Molodensky's problem. Columbus: Department of Geodetic Science, Ohio State University, 1968, 46 p. Relatório científico.

MORITZ, H. Advanced physical geodesy. Karlsruhe: Wichmann, 1980.

PAVLIS, N. K.; SIMON, H. A.; STEVE, K. C.; JOHN, F. K. An Earth Gravitational Model to Degree 2160. In: EGU General Assembly, 2008, Vienna. Disponível em: <http://www.dgfi.badw.de/typo3_mt/fileadmin/ 2kolloquium_muc/2008-10-08/Bosch/EGM2008.pdf >. Acesso em: 9/2/2010.

PICK, M.; PÍCHA, J.; VYSKOČIL, V. Theory of the Earth's gravity field. Amsterdam: Elsevier, 1973.

RODRIGUEZ, E.; MORRIS, C. S.; BELZ, J. E.; CHAPIN, E. C.; MARTIN, J. M.; DAFFER, W.; HENSLEY, S. An assessment of the SRTM topographic. Technical Report JPL D-31639, Jet Propulsion Laboratory, Pasadena, California, 2005, p. 143.

ROY, K. K. Potential theory in applied geophysics. Calcutta: Springer, 2008.

SÁ, N. C. de. Observações GPS sobre referências de nível. Instituto Astronômico e Geofísico, Universidade de São Paulo. São Paulo. Comunicação pessoal em 2007, 20-?

SANCHÉZ, L. Determinación de la superfície vertical de referencia para Colombia. 106 f. Tese - Geodésia Física e Teórica, Universidade Técnica de Dresden, Dresden, 2003.

SANSÒ, F. Molodensky's problem in gravity space: A review of the first results. Bulletin Géodésique, v. 52, n. 1, p. 59-70, 1978.

SHEPPERD, S. W. A recursive algorithm for evaluating Molodenskii-type truncation error coefficients at altitude. Bulletin Géodésique, v. 56, n. 2, p. 95$105,1982$.

SIDERIS, M. G. Rigorous gravimetric terrain modelling using Molodensky's operator. Manuscripta Geodaetica, v. 15, n. 2, p. 97-106, 1990.

SIDERIS, M. G.; SCHWARZ, K. P. Solving Molodensky's series by fast Fourier transform techniques. Bulletin Géodésique, v. 60, n. 1, p. 51-63, 1986.

TORGE, W. Geodesy. Berlin: Gruyter, 2001.

VAL'KO, M.; MOJZES, M.; JANÁK, J.; PAPCO, J. Comparison of two different solutions to Molodensky's G1 term. Studia Geophysica et Geodaetica, v. 52, n. 1, p. 71-86, 2008.

VELKOBORSKY, P. On the solution of the Molodenskii boundary value problem using successive approximations. Studia Geophysica et Geodaetica, v. 13, n. 3, 
p. 259-266, 1969.

YEREMEEV, V. F.; YURKINA, M. I. Fundamental equations of Molodenskii's theory for the gravitaional reference field. Studia Geophysica et Geodaetica, v. 18, n. 1, p. 8-18, 1974.

YEREMEEV, V. F.; YURKINA, M. I. On the conditions of solvability of Molodenskii's problem and a transformation of his integral equation. Studia Geophysica et Geodaetica, v. 14, n. 2, p. 126-135, 1970.

(Recebido em março de 2010. Aceito em setembro de 2010.) 